\title{
Propuesta educativa metodológica aplicada a través del comics por una construcción de paz
}

\author{
Methodological educational proposal applied through comics for \\ a construction of peace
}

\section{Recibido: 12-11-2021 | Aceptado: 10-12-2021}

\section{Resumen}

Las comunidades académicas escolares nacionales e internacionales son conscientes de las problemáticas psicosociales por las que afrontan las escuelas diariamente. Es por tanto la necesidad de generar planes y programas educativos, pedagógicos creativos e innovadores que sean atractivos a los estudiantes para de esta manera fortalecer nuevos aprendizajes en competencias que faciliten las buenas relaciones interpersonales y las disminuciones de los conflictos. El presente estudio es resultado de una aplicación de investigación aplicada que tiene como objetivo construir representaciones dialógicas a través del comic como medio pedagógico para promover las construcciones de paz en los contextos escolares. El estudio es de tipo cualitativo, del método representativo de la Investigación-Acción-Participación donde las personas observan y estudian sus propias realidades con fines de concientizarse de sus propios problemas sociales y de resolverlos. La población participante fueron 30 niños y niñas de la Institución Educativa "San José" de la ciudad de Barranquilla-Colombia. En los resultados se observó a cada uno de los niños en su libre naturalidad ejerciendo un rol mediador, en que a través del diálogo, con el uso adecuado del lenguaje, les permitió la diversidad de gustos, formas de comprender el conflicto y construir paz. Como conclusiones esta propuesta de cultura de paz con aplicación pedagógica creativa del comic, le apuesta a la educación para la paz, cultura de paz para ayudar a construir valores mediante acciones humanas que permita la involucración y participación de todos los niños y niñas, para ayudarlos a construir, fortalecerlos en valores y cambios de actitud.

Cómo citar

Albor-Chadid, L. Propuesta educativa metodológica aplicada a través del comics por una construcción de paz. MSC Métodos De Solución De Conflictos, 2(2). Recuperado a partir de https://revistamsc.uanl.mx/index.php/m/ article/view/18 
Palabras clave: Comics, Diálogo, Pedagogía, Construcción De Paz.

\begin{abstract}
The national and international academic school communities are aware of the psychosocial problemas that schools face on a daily basis. It is therefore the need to generate creative and innovative educational, pedagogical plans and programs that are attractive to students in order to strengthen new learning in competencies that facilitate good interpersonal relationships and decrease conflicts. Thus, the present study is the result of an applied research application that aims to build dialogic representations through the comic as a pedagogical means to promote peacebuilding in school contexts. The study is of a qualitative type, of the representative method of Research-Action-Participation where people observe and study their own realities in order to become aware of their own social problems and to solve them. The participating population was 30 boys and girls from the Educational Institution "San José" in the city of Barranquilla-Colombia. In the results, each one of the children was observed in their free naturalness, exercising a mediating role, in which, through dialogue, with the appropriate use of language, they allowed a diversity of tastes, ways of understanding the conflict and building peace. As conclusions, this proposal for a culture of peace with creative pedagogical application of the comic, bets on education for peace, a culture of peace to help build values through human actions that allow the involvement and participation of all children, to help them to build, strengthen them in values and attitude changes.
\end{abstract}

Keywords: Comics, Research-Action-Participation, Pedagogy, Peacebuilding.

\section{INTRODUCCIÓN}

El Ministerio de Educación Nacional República de Colombia, a través de la Constitución Política de 1991, la Ley General de Educación 115 de 1994 y los diversos convenios internacionales suscritos por Colombia, reconocen la importancia de los derechos humanos como objetivo fundamental de la educación dentro del marco de la formación ciudadana como son el respeto, la defensa y la promoción de los derechos humanos.

Es así como se promulga por las competencias ciudadanas que se enmarcan en la perspectiva de derechos y brindan herra- mientas básicas para que cada persona pueda respetar, defender y promover los derechos fundamentales, relacionándolos con las situaciones de la vida cotidiana en las que éstos pueden ser vulnerados, tanto por las propias acciones, como por las acciones de otros. Por tanto, las competencias ciudadanas representan las habilidades y los conocimientos necesarios para construir convivencia, participar democráticamente y valorar el pluralismo (Ministerio de Educación Nacional, República de Colombia, 2019).

A pesar que el Ministerio de Educación Nacional en Colombia ha procurado por mante- 
ner y operacionalizar las normas, leyes, principios que procuren el desarrollo humano y social de toda una comunidad escolar académica, aún persiste la divergencia entre lo real y la norma, puesto que actualmente las situaciones de conflictos que se presentan en las escuelas tales como los estudiantes señala que el conflicto está lastimando a compañeros mental y físicamente, así como también lo describen como algo negativo que es inaceptable, desagradable y repulsivo. Lo califican como acciones violentas físicas de un estudiante a otro. A nivel mental piensan que el conflicto es una situación que perturba y que es algo en lo que nada se puede hacer con consecuencias que puede estar afectando sus valores.

Es de importancia anotar que ante lo adverso de construir paz en las instituciones educativas se encuentra la violencia escolar, es por tanto que actualmente se están desarrollando múltiples investigaciones con resultados que evidencian que las causas que más generan los conflictos en las escuelas son el abuso de sustancias, los problemas económicos, políticos y sociales, el género, la adaptación social, las relaciones entre pares y maestros, la adaptación social y los factores psicológicos, siendo América Latina la región que más problemáticas presentan, debido a las circunstancias que enfrentan de problemas de violencia en general (Rodríguez-Burgos et al 2020).

Es así como el Estado Colombiano a la luz de generar relaciones directas entre la construcción de paz y el fortalecimiento de una idea de Estado, se comprende como una institución que debe crear y garantizar las condiciones para la paz a través del artículo 22 de la Constitución Política de Colombia que estipula que "La paz es un derecho y un deber de obligatorio cumplimiento" donde se comprende como un deber en tanto involucra a toda la ciudadanía colombiana desde su aspecto más formal, relacionado con el Estado y sus instituciones, hasta el más emergente, relacionado con lo social y las demandas de la sociedad civil (Grajales et al 2015).

Con esta medida implementada por el Ministerio de Educación para el desarrollo humano de los estudiantes y atendiendo a las necesidades de las diferentes problemáticas sociales que se presentan en las instituciones educativas principalmente los conflictos escolares y a su formación biopsicosocial, se visualiza la necesidad de orientar unas prácticas pedagógicas con objetivos motivados a aplicar una metodología con estrategias interpretativas que muestre el significado subjetivo y que esté unido a unas respuestas conductuales en búsqueda de comprender las respuestas sociales y búsquedas de alternativas de solución que aminoren los conflictos. Es así que este estudio tiene como objetivo principal construir representaciones dialógicas a través del comic como medio pedagógico para promover las construcciones de paz en los contextos escolares.

Por tanto, se presenta El comic en Iberoamérica como el producto de un conjunto de tradiciones editoriales y culturales diversas que, ahora en el contexto de un renovado interés por la historieta, plantea propuestas y miradas cada vez más difíciles de ignorar 
por parte del establecimiento cultural (Correa et al 2010).

De igual manera Correa et al (2010) afirman que el comic hace evidente el hecho de que cuanto más fluida es la comunicación con un público amplio, la producción secuencial es más un espacio de reflexión colectiva dispuesto a abordar los temas más complejos con la mayor seriedad y respeto (pág. 50).

Por cuanto la propuesta del comic representa atributos que atribuye manifestadas en competencias llevadas a las acciones con las reflexiones y significados propios de cada persona. Cabe decir también que tiene una facilidad para indagar interpretaciones que permite considerar y revisar críticamente las autorrepresentaciones propias de cada individuo y su forma de interactuar individual y socialmente.

Esta propuesta de cultura de paz con aplicación pedagógica creativa del comics, le apuesta a una educación para la paz con fines de aumentar las herramientas de intervención a la ciudadanía para ayudar a construir valores y actitudes tales como la justicia, la libertad, la libre expresión de pensamientos, el respeto, la solidaridad mediante acciones humanas que permita la involucración y participación de todos los niños y niñas, para orientarlos a construir y fortalecerlos en valores y nuevos cambios de actitud para afrontar situaciones de conflictos.

Como descripción y lugar de los acontecimientos donde se desarrolla la propuesta, este documento es fruto del trabajo de te- sis de grado que se ha estado construyendo con el Doctorado en Métodos Alternos en Solución de Conflictos en convenio con la Universidad de Nuevo León - Monterrey - México. Esta experiencia educativa pedagógica reflexiva se está construyendo con niños y niñas de la Institución Educativa "San José" de la ciudad de Barranquilla - Colombia, desde sus cotidianidades académicas. Hecha la descripción anterior, se continúa estructurando la fundamentación teórica puesta a disposición que nos revelará los principios, supuestos para ser evidenciados en la práctica con la participación de la población.

\section{LITERATURA REVISADA}

Carr \& Kemmis (1988) afirman que las estrategias pedagógicas educativas sociales podrían revelar en el plano social estructural el carácter ideológico de la vida de un grupo, demostrando el modo en que los procesos sociales como el lenguaje y otros atributos de producción de medios de comunicación, así como de reproducción cultural, configuran las experiencias del mundo social, así como también buscan formas para transformar y mejorar el proceso de enseñanza y aprendizaje.

Asimismo, tal como afirma Weber (1977) que el hombre es un animal inserto en tramas de significados que él mismo ha tejido donde la cultura es esa urdimbre y la que el análisis de la cultura ha de ser, por lo tanto, no una ciencia experimental en busca de leyes, sino una ciencia interpretativa en busca de significados. 
Así mismo complementando lo anterior, se especifica la importancia de la acción-comunicativa, donde Habermas (1989) expone la realidad social como una construcción del mundo de la vida cotidiana, que resulta de los "rendimientos interpretativos" de las personas directamente implicados; es decir concebir la comprensión como el modo privilegiado de experiencia.

Así mismo Habermas (1989) expone la lingüística y las acciones social como pretensiones de validez de modo similar a los actos de habla con enunciado y enunciación, es decir constituyen medios de acciones comunicativas en que el individuo aprende estrategias de afrontamiento para confrontar su contexto actual en casos en que se tornen problemas, conflictos y en las que se hace menester llegar a un acuerdo a través del diálogo argumentativo donde se reconocen simultáneamente a algo en el mundo objetivo, en el mundo social y en el mundo subjetivo, para negociar definiciones de la situación que puedan ser compartidas por todos (pág. 143).

Desde esta propuesta se destaca la cultura y educación para la paz como medios de programas de construcciones de paz en el marco de la mediación educativa como concepto impulsado por la Organización de las Naciones Unidas para la Educación, la Ciencia y la Cultura (UNESCO) que busca que los Estados miembros adapten y evolucionen sus políticas públicas educativas, en la norma y en la aplicación, a fin de asegurar la formación en valores, derechos humanos, paz, participación democrática, tolerancia, no violencia y entendimiento intercultural, englobado en el contexto internacional de la educación para la paz (Citado por Vázquez, 2015).

La cultura de paz es definida por la Organización de las Naciones Unidas para la Educación, la Ciencia y la Cultura - UNESCO, como aquel conjunto de valores, actitudes, tradiciones, comportamientos y estilos de vida basados en el respeto a la vida, el fin de la violencia y la promoción y la práctica de la no-violencia por medio de la educación, el diálogo y la cooperación, el respeto pleno y la promoción de todos los derechos humanos y las libertades fundamentales; el compromiso con el arreglo pacífico de los conflictos y el respeto y el fomento de la igualdad de derechos y oportunidades de mujeres y hombres además que encuentra en la mediación una herramienta eficaz para el logro de estos objetivos (Citado por Vázquez, 2015).

Cabe señalar que Velasco Suárez, et al (2012) sostienen que los principios de la Educación para la Paz están basados en la legitimidad y en la justicia moral que podrá dar lugar a un nuevo orden de equilibrio y convivencia responsable en el mundo ( $\mathrm{Ci}$ tado por Cabello-Tijerina \& Vázquez Gutiérrez, 2018).

De lo anteriormente expuesto, se nombra la pedagogía como recurso facilitador para alcanzar estos atributos de formación integral, es así como Muñoz \& López Martínez (2004) afirman que:

La Educación para la Paz es también nombrada pedagogía de la paz que nace como estudio de los comporta- 
mientos agresivos y violentos en los seres humanos y apuesta por unas formas de socialización y educación diferentes que hicieran a las personas más libres, responsables y creativas.

Es así como el rigor de este discurso nos permite observar los principios de la educación para la paz tale como afirman Hoyos \& Vargas (1997):

1. El nivel del reconocimiento del otro $y$ de las diferencias, de la multiplicidad de perspectivas, puntos de vista, paradigmas y culturas siendo finalmente el momento comunicativo, dado que comprender al otro no nos obliga a estar de acuerdo con él, pero sí hace posible el que lo estemos o el que sepamos por qué no lo podemos estar.

2. Pretender la intención manifiesta en el lenguaje y expresión libre en el lenguaje tal cual como se expresa que se refiere a un mundo subjetivo que se va reproduciendo en procesos de formación de la persona. En este mundo subjetivo habitan las personas desde el punto de vista de sus diferentes formas de interactuar socialmente es decir desde su autocomprensión e identidad.

Con base en lo anterior y la importancia que tiene la educación aplicando la pedagogía como medio para construir la paz, éstas se estructuran como fortalezas para alcanzar los aprendizajes, para constatar la veracidad de los saberes evidenciados en las realidades, el aumento del pensamiento crítico, la comunidad estudiantil como lo interpreta, como determinaría para la toma de decisiones y resuelve conflictos.
Además, también se destaca en estos procesos, la eficacia y autodeterminación de aplicar modelo de innovación pedagógica en las escuelas colombianas, lo que estimula la autoeficacia y motivación académica, el apoyo social, la afiliación escolar, así como nuevos reforzadores tanto cognitivos, emocionales y conductuales por la disminución de la incidencia de la violencia escolar (Albor-Chadid, et al 2020).

Entonces es de señalar los principios de la educación para la paz que Palos Rodríguez (2010) señala:

1. Enseñar y aprender a resolver conflictos. Educar para la paz es una forma particular de educar en valores. Cuando educamos, consciente o inconscientemente se está transmitiendo una escala de valores.

2. Ayudar a construir unos valores y actitudes determinados tales como la justicia, libertad, cooperación, respeto, solidaridad, actitud crítica, el compromiso, la autonomía, el diálogo, la participación. Al mismo tiempo se cuestionan los valores que son contrarios a la paz como la discriminación, la intolerancia, la violencia, el etnocentrismo, la indiferencia, el conformismo.

3. Educación desde y para la acción. No se trata de educar para inhibir la iniciativa y el interés sino para encauzar la actividad y el espíritu combativo hacia la consecución de resultados útiles a la sociedad. Se trata de participar en construcciones de paz. (Citado por Vázquez, 2015).

Sin embargo, no se debería desconocer que dentro de las interacciones sociales en que vive el ser humano los efectos de las socia- 
lizaciones, diferencias de estilos de vida y crianza predisponen a las situaciones de conflictos.

Entonces se podría afirmar que, desde las construcciones de paz, la educación para la paz constituye una línea de intervención de cambio social que desde la construcción de paz versus educación para la paz hacen referencia de comprender que la construcción de paz no es ausencia del conflicto. Por el contrario, se asume que el conflicto es consustancial a las relaciones entre los seres humanos, por tanto, es un elemento fundamental para repensar la paz y las variadas formas en que los seres humanos lo resuelven bajo estrategias no violentas, como la creación de ambientes educativos de sana convivencia y de reconocimiento de la diversidad y la diferencia (Grajales, et al, 2015).

Es de reconocer entonces la emanación de las políticas públicas para implementar planes, programas, proyectos que promulguen soluciones a las situaciones de conflictos que surgen en las instituciones escolares con fines de promover la cultura de la convivencia y soluciones pacíficas a los conflictos (Miranda-Medina \& Gorjón, 2018, como se citó en Enamorado et al 2020).

Por lo anterior, es necesario resaltar la importancia de la mediación educativa en los contextos escolares como afirma Vázquez (2015), como una estrategia de amplia acción dentro de la contextualización de la educación para la paz y a su vez en dirección objetiva de una educación integral, una formación entendida como un todo que se compone de una gran diversidad de elementos individuales, recursos materiales, económicos, sociales y familiares. (pág. 59). Con base en lo anterior, la mediación como método alterno de solución de conflictos, constituye herramientas de comunicación, de diálogos de saberes con estructuras que facilitan el intercambio natural de opiniones, de las realidades y que a través de la lingüística se expresan símbolos, representaciones mentales en relación con un ser humano lleno de historias y experiencias sociales.

Es de importancia anotar como afirma Pérez de Guzmán Puya (2009) que, con los métodos de las mediaciones aplicadas en los contextos escolares, el paradigma constructivista es visible ya que se respetan los sentimientos e intereses de los implicados y contribuye a la formación individual y grupal (Citado por Vázquez, 2015).

Ahora continuando con lo anterior y la importancia del aprendizaje constructivista en la forma de instruir la paz, Riveros (2009) y Espinoza (2017) señalan que se requiere de una predisposición para aprender significativamente y para efectuar el esfuerzo mental que eso supone. De aquí la importancia de que la función principal del profesor sea que sus alumnos aprendan de manera significativa. En este sentido se retoma lo manifestado por Pérez Gómez (2008) y Pérez Sánchez y Beltrán Llera (2014) respecto a que la motivación es el motor del aprendizaje que se encuentra influida por las emociones, las creencias, los intereses y los valores. (Citado por Carranza Alcantar, Caldera Montes, 2019).

La escuela es actualmente un sistema social que debe responder a las exigencias pro- 
pias de una cultura, pero ante la exigencia de unas crisis de adaptación de todo un sistema académico, el logro de metas, se presentan los episodios de conflictos escolares evidenciados en las conductas violentas, es por tanto que los mecanismos de constante innovación pedagógica permite a la comunidad académica a utilizar herramientas creativas, didácticas para trabajar desde la academia los conflictos sociales en las escuelas (Albor-Chadid, 2019).

Por tanto, el interés de lo anterior se centra en aumentar la eficacia de la mediación, la formación del mediador y el análisis de los programas de mediación escolar, en aumentar la convivencia escolar y la promoción de la mediación escolar en políticas públicas con aplicación de estrategias innovadoras en pedagogía (Miranda-Medina et al 2020).

Es así como el cómic como mediación pedagógica en su estructura narrativa cumple un propósito esencial que es la de "distraer", es por tanto que debido a las necesidades y al prestigio social se ha implementado en las aulas de clase para motivar a los estudiantes a aprender y para hacer uso de éste como una mediación pedagógica facilitando el acto educativo, para los docentes entrevistados consideran que "El cómic siempre ha sido una herramienta muy valiosa y subsidiaria de las disciplinas para transmitir mensajes, conocimientos y enseñanzas, a través de las imágenes y de los textos que son llamativos para los estudiantes" (Oliveros Zambrano, Pulido González, \& Rincón Pinilla, 2017).
El propósito del cómic se afirmaría que acentúa nuevas aperturas de comunicación humana que nos muestra posibilidades de interpretar, comprender las realidades humanadas de otras personas a la vez ser utilizado como mediación pedagógica donde instruye para aumentar los canales de comunicación, el fomento por liderazgo en los grupos, la toma de decisión en grupo, la facilidad para mediar situaciones de conflictos.

Es por tanto que el comic permite ser utilizado como técnica de doble vía, por una parte, como herramienta pedagógica de sentido de autorreflexión y por otra parte con posibilidades de medir el conocimiento científico ya que permite utilizarlo con estructuras metodológicas aplicadas y observar los cambios de percepción de realidad y actitudinales. Es así como Latorre, (2016) afirma que:

La facilidad del cómic para genera estructuras narrativas cercanas al contexto de los estudiantes es pertinente preguntarse sobre el tipo de enseñanza que se busca por lo tanto es relevante comprender este aspecto por su significatividad entendida como el aprendizaje de las temáticas tanto discursivas como históricas con posibilidad de aplicarse de manera práctica con estructuras lógicas que permita un razonamiento estructurado por parte de los estudiantes y por su funcionalidad, que lo aprendido modifique activamente su futura relación con la temática que sea "un aprendizaje extrapolable $\mathrm{o}$ ampliable" (Citado por Vargas Gómez, 2019). 


\section{MÉTODO}

La construcción de la propuesta con base en competencias ciudadanas por una construcción de paz implicó procesos de interpretación con la aplicación del uso del comics como estrategia pedagógica que permitieran reflexiones e interpretaciones con relación de la teoría con la práctica y aplicación del método representativo de la Investigación-Acción-Participación donde las personas observan y estudian sus propias realidades con fines de concientizarse de sus propias problemas sociales y actuar en toma de decisiones de acciones o transformación social. El estudio es de tipo cualitativo ya que se necesitó explorar las percepciones, actitudes de los individuos. Se adoptó un postulado constructivista con la participación de las personas comprometidas en el desarrollo del programa.

\subsection{Participantes}

Los participantes fueron 12 niñas y 18 niños para un total de 30 estudiantes del Grado Sexto en edades entre los 10 y 13 años de edad de la Institución Educativa Distrital "San José" ubicado en la ciudad de Barranquilla-Colombia.

\subsection{Instrumento}

Se utilizó una entrevista semiestructurada para la recolección de datos. La guía de la entrevista se aplicó en tres talleres interactivos que incluyeron preguntas para ser indagadas a través del comic con instrucciones pedagógicas que hicieron referencia a la convivencia y al diálogo entre compañeros como mediación para la resolución de conflictos y la construcción de paz en la escuela.

\subsection{Procedimiento}

En cada sesión de los talleres se instruía a los participantes con una pregunta de orientación que luego se complementaba con subpreguntas para mayor razonamiento, interpretación y flexibilidad para medir las situaciones de conflictos indagadas a través del comic. Además, se les solicitó a los participantes comentarios adicionales acerca de la creatividad, motivación por construir escenarios sobre el diseño de su comic.

Para el análisis e interpretación de la información cualitativa aplicada a través del uso del comic se determinó una categoría principal que permitiera atender al objeto de estudio. En la categoría se especifica dos subcategorías con definiciones para ampliar los atributos naturales del individuo en su construcción para promover construcciones de paz. Además, se presenta la estructura de la guía de entrevista semiestructurada aplicada. 
Tabla 1: Categorías-Subcategorías

\begin{tabular}{|l|l|l|l|}
\hline \multicolumn{1}{|c|}{ Categoría } & \multicolumn{1}{|c|}{ Subcategoría } & \multicolumn{1}{c|}{ Definición } & \multicolumn{1}{c|}{ Guía de preguntas } \\
\hline \multirow{5}{*}{$\begin{array}{l}\text { Representaciones } \\
\text { dialógicas }\end{array}$} & $\begin{array}{l}\text { Relación entre el } \\
\text { lenguaje y la men- } \\
\text { te humana. }\end{array}$ & $\begin{array}{l}\text { El lenguaje constituye } \\
\text { el instrumento dócil } \\
\text { y transparente de } \\
\text { la expresión de } \\
\text { representaciones } \\
\text { producidas por la mente } \\
\text { a través del dibujo. }\end{array}$ & $\begin{array}{l}\text { ¿Quiénes son las partes del } \\
\text { conflo? } \\
\text { ¿Piensas que tu comic, } \\
\text { facilitará la comunicación } \\
\text { con tus compañeros? } \\
\text { ¿Es el diálogo un medio } \\
\text { que facilita resolver } \\
\text { problemas entre las } \\
\text { personas? }\end{array}$ \\
\cline { 2 - 4 } & \multirow{2}{*}{$\begin{array}{l}\text { Participación } \\
\text { práctica de las } \\
\text { personas. }\end{array}$} & $\begin{array}{l}\text { Componente natural } \\
\text { del individuo en que } \\
\text { asume consciencia de } \\
\text { su espacio, posición, } \\
\text { integrándose en } \\
\text { dinámicas grupales a } \\
\text { través de la práctica. }\end{array}$ & $\begin{array}{l}\text { ¿De qué forma te } \\
\text { relacionarías con tus } \\
\text { compañeros para construir } \\
\text { paz? } \\
\text { ¿Piensas que construir paz } \\
\text { te hace sentir mejor? }\end{array}$ \\
\hline
\end{tabular}

Fuente: Análisis propio.

De acuerdo a lo anterior y teniendo en cuenta la estructura organizada metodológica aplicada para trabajar la estrategia pedagógica del comic y su importancia en las construcciones de paz escolares, se empezó la actividad motivando a los participantes a participar pensando en una lluvia de ideas acerca de acciones para resolver un conflicto. En esta actividad cada niño representó una historia con carácter lúdico a través del dibujo, acerca de sus opiniones en condiciones de libertad, libre expresión acerca de mediar situaciones de conflicto. Luego se reunieron en grupos, cada uno expresó, dialogó, debatieron, tomaron la palabra, expresaron sus ideas acerca de comic, reflexionaban acerca de lo que dialogaban y como cada quien aportaba reglas para promover construcciones de paz.

\section{RESULTADOS}

Es de destacar la importancia de la escuela como sistema de relaciones grupales donde se debería actualizar en utilizar herramientas creativas, didácticas para trabajar desde la academia, el diálogo como una herramienta natural, lingüística para construir sistemas de paz y así disminuir los conflictos sociales en las escuelas.

Por lo anterior, la investigación aplicada aporta resultados apreciables que permiten la mejora en la sociedad (Miranda-Medina \& Rodríguez-Burgos, 2017) con la aplicación de diferentes métodos cualitativos y cuantitativos (Sáenz \& Rodríguez-Burgos, 2010; Rodríguez-Burgos \& Gorjón, 2014; Rodríguez-Bur- 
gos, 2012) con expectativas de transferir ese conocimiento a las instituciones educativas en procura de mejorar la calidad de la educación y el desarrollo de los estudiantes así como también el aporte de herramientas didácticas y estratégicas para mejorar el sistema educativo (Citado por Rodríguez-Burgos $\&$ Miranda-Medina 2020).
A continuación, se presentan los resultados del comic creado y representado socialmente con la participación de los niños y niñas del Grado Sexto de la Institución Educativa Distrital "San José".

\section{Figura 1: Representación dialógica del conflicto, Subcategoría relación entre el lenguaje y la mente humana.}

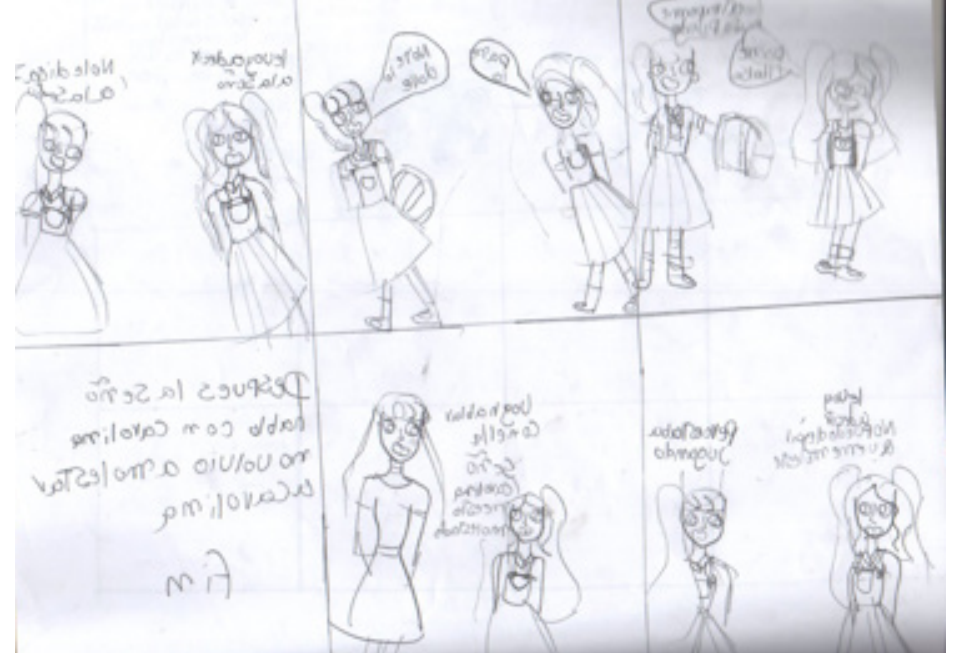

Fuente: Elaboración propia.

Como novedad e innovación, las instrucciones suministradas para la creatividad y uso del comic, recibió anotaciones muy positivas. Los participantes mostraron autonomía, atentos, espontáneos y divertidos en cada uno de sus diseños. Expresaron opiniones como: "Me gustó todo, sobre todo, representar a un súper héroe llamado "Antibullin" con poderes para resolver los conflictos en una aventura".

En la subcategoría relación entre el lenguaje y la mente humana, surgió una subcategoría denominada "Diálogo" donde se observó cómo cada uno de los niños naturalmente a través del lenguaje en su ejercicio de narrar su comic, ejercían un rol mediador entre un compañero y otro; es decir, se generó el juego de preguntas y de respuestas que facilitaba la interacción grupal, así como la comprensión de los dibujos en sus contextos culturales. La interacción simple de hablar y de exponer los símbolos, signos, frases o enunciados representados en el comic, les permitió poder reflexionar acerca de sus propios mundos y el de los demás.

Es de señalar que, en las entrevistas, 22 participantes de 30 , en la subcategoría "parti- 
cipación práctica de las personas" narraron aspectos relacionados con la mediación. Hablaron que se les hacía más fácil construir paz a través de la representación de un superhéroe que tiene la intención de manifestar algo, sobre algo que hay que solucionar en instrucciones estilo comic.

Es de anotar que desde el componente natural del individuo en participar de actividades en las que se apoyó de sus habilidades lingüísticas a través del dibujo, los motivo a reforzarse en sus áreas cognitivas, emocionales y conductuales en disminuir los conflictos y malos entendidos en frases o enunciados como: "siento que hice mal, voy a hacer las paces con ese compañero", Amigo, discúlpame. Te disculpo, somos amigos y no tuvimos que pelear".

De acuerdo a lo anterior, como afirma Palos Rodríguez (2010), los niños construyen valores, cuestionan los valores contrarios a la paz, así como también afirma Riveros (2009) y Espinoza (2017) que se requiere de una predisposición para aprender significativamente y para efectuar el esfuerzo mental que la actividad supone. En la interacción social aceptan y obedecen la dinámica de manera consciente, desinhibida que les ayuda a crecer integralmente a resolver conflictos a parte que los mantenía concentrados y divertidos en la experiencia pedagógica. Además, como afirma Pérez de Guzmán Puya (2009), en estos episodios de creatividad, diálogos, encuentros culturales, los niños construyen sus formaciones individuales y grupales sensibles al espíritu y respeto por las emociones a partir de su comunicación verbal y no verbal, así como las condiciones adecuadas para afrontar los conflictos con acciones inherentes de construir sanas convivencias de paz.



Fuente: Elaboración propia. 
Las representaciones dialógicas que se describe en la figura 2, refleja como los cuadros ocupan un espacio en sus mundos reales, sus posibilidades ilimitadas de manifestaciones acerca de sus percepciones, proyectando a través del dibujo sus actitudes, pensamientos, sentimientos, expresión de valores y formas conductuales que están en sus procesos e intenciones de construcción de paz y de responder a una forma particular construir formas adecuadas de convivencia. Además, también como afirma Palos Rodríguez (2010), la actividad los encauza hacia metas, resultados que les puedan ser útiles tanto a los niños como a la sociedad, así como también desde los aportes de Habermas (1989), quien afirma que las realidades sociales se construyen del mundo de la vida cotidiana, que resulta de los "rendimientos interpretativos" de las personas directamente implicados; es decir concebir la comprensión como el modo privilegiado de experiencia.

Por lo anterior, es importante anotar que las formas de la representación dialógica determinadas por los estudiantes evidenciados en la figura 2 en su comic, en 21 entrevistas de 30 , señalan la presencia diaria y normal de los episodios de violencia, el maltrato físico y verbal en la escuela. Pero lo destacable consistió en que aquellos participantes en que su discurso era bajo, se motivaron a expresar algo, a pronunciar cualidades del contenido secuencial en tiempo, espacio de las acciones de sus personajes creativos del comic para mediar una situación de conflicto.

Lo importante de la representación dialógica del dibujo expresado en el comic, es que el estudiante explora la situación del conflic- to, intenta descubrir desde sus propias habilidades naturales como es la comunicación, el diálogo, el medio natural del habla, que le permita solucionar el problema, así como también muestra sensibilidad y determinación en encontrar alivio emocional y medios alternativos para construir ambiente de armonía y paz.

\section{CONCLUSIONES}

Esta propuesta de cultura de paz con aplicación pedagógica creativa del comic, le apuesta a la educación para la ciudadanía para ayudar a construir valores y actitudes tales como la justicia, la libertad, la libre expresión de pensamientos, el respeto, la solidaridad mediante acciones humanas que permita la involucración y participación de todos los niños y niñas, para ayudarlos a construir y fortalecerlos en valores y nuevos cambios de actitud.

Así mismo posibilita los cambios mentales en cuanto a la desinhibición de sus propias creencias a través del dibujo y así mismo comparándolos y evidenciarlos en sus propias realidades.

Es de anotar que, a través de la estrategia didáctica, los niños a través del diálogo focalizan un problema, lo analizan, lo interpretan y tratan de desplazar el problema a situaciones en que ellos pueden resolverlos, además que se fomentan las habilidades discursivas, el derecho a hablar como un vínculo moral (relaciones interpersonales con el compañero), así como también el fomento por la igualdad de género para fortalecer la mediación entre compañeros. 
La construcción de la propuesta pedagógica por una construcción de paz, implica procesos de reflexión, interpretación que relacione la teoría con la práctica a través de la praxis con criterios de organización y orden para propiciar ambientes de mediación.

La estrategia pedagógica dirigida a una cultura de paz tiene un enfoque humanista y social que direcciona la exploración de los valores sociales con objetivos de fortalecerlos en sus relaciones interpersonales.

La cultura de paz a través de la mediación, articulan intereses de diversos tipos de saberes en que exploran sus propias culturas, costumbres, creencias, prejuicios, así como comprensión de sus propias historias.

Es por tanto que esta estrategia lúdica pedagógica con objetivos de resolución de conflictos, parte de la participación de sus integrantes, al sentido de autorreflexiones, en el uso de la comunicación a través de la lingüística por las oportunidades de hablar, de dialogar, de apostar por nuevas reconstrucciones del sentido de la vida.

Es de señalar que los momentos pedagógicos dirigidos hacía las construcciones de paz constituyeron unas dinámicas de relaciones horizontales con los participantes, facilitando el sentido de lo social, construcciones de lo social y donde el lenguaje y la comunicación tenían un sentido de reproducción racional de sus propias historias.

La aplicación del comic como un instrumento pedagógico en que el individuo expresa en el significados subjetivos a través del dibujo donde descubre e interpreta un conjunto de actitudes que le dan construcciones de sentido y enseñan a aprender a resolver conflictos.

Así también desde las narraciones que propicia en la que se revele la posibilidad de interpretar realidades y métodos alternos (mediación, conciliación), en la que valoren de manera espontánea sus desarrollos, autoperciban su formación, reinterpreten sus propias realidades y así modificar sus actos. Ayuda a describir y descubrir las competencias, creativas e innovadoras que cada niño tenga y pueda aportar hacia sus propias construcciones integrales por las construcciones de paz.

Asumir la estrategia como un desafío la técnica didáctica del comic como un instrumento para generar investigaciones educativas en búsqueda de aumentar las estrategias de enseñanzas para reducir los conflictos escolares y las construcciones de paz.

Cabe señalar que la efectividad de la aplicación de la técnica del comic depende de la debida utilización metodológica pertinente por parte del docente con sentido de orientar e influenciar en el estudiante en el nuevo aprendizaje y a partir de allí, explorar sus habilidades, competencias de construcciones de identidad, así como el de fortalecer sistemas de construcción de paz.

\section{TRABAJOS CITADOS}

Albor-Chadid, L. I. (2019). Comics, medio innovador, creativo pedagógico para la resolución de conflictos escolares. Eirene Estudios de Paz y Conflictos, (2). 
Albor-Chadid, L. I., Filut, D., Valero-Díaz, B., \& Rodríguez-Burgos, K. (2020). Propuesta de modelo de innovación pedagógica para disminuir la deserción escolar en Colombia desde la experiencia de Israel. Revista de Estudos de Literatura, Cultura e Alteridade-Igarapé, 12(4).

Cabello-Tijerina, P. \& Vázquez Gutiérrez, R. (2018). “ Cultura y Educación para la Paz. Una perspectiva transversal/ Culture and Education for Peace. A Transversal Perspective, 2018. Ciudad de México: Tirant Lo Blanch.

Carr, W \& Kemmis, S. (1988). El planteamiento interpretativo de la teoría y la práctica educativa. Teoría crítica de la enseñanza. Barcelona, editorial Martínez Roca.

Carranza Alcántar M; Caldera Montes, J. (2019). Percepción de los Estudiantes sobre el Aprendizaje Significativo y Estrategias de Enseñanza en el Blended Learning. REICE. Revista Iberoamericana sobre Calidad, Eficacia y Cambio en Educación, [S.I.], v. 16, n. 1, ene. 2018. ISSN 1696-4713. Disponible en: 〈https://revistas.uam. es/index.php/reice/article/view/9031/9282〉. Fecha de acceso: 29 oct. 2019 doi:http://dx.doi.org/10.15366/reice2018.16.1.005.

Correa, J., Guerra, P., \& Vergueiro, W. (2010). El cómic, invitado a la biblioteca pública (No. 20). e-libro, Corp.

Enamorado-Estrada, J., Morcote, O., Bayuelo-Schoonewolff, P. \& Rivas-Castillo, C. (2020). School coexistence educational public policies to prevent school violence. En K. Rodríguez-Burgos (coord.), School violence radiography (pág. 45-81). Barranquilla: Ediciones Universidad Simón Bolívar.

Espinoza, J. (2017). Los recursos didácticos y el aprendizaje significativo. Espirales. Revista Multidisciplinaria de Investigación, 2, 33-38.

Grajales, C. V. E., Ospina, J. B., Suárez, N. A. M., Meléndez, L. G., \& Beltrán, L. A. C. (2015). Contribuciones de la institución educativa al postconflicto: Humanizarte, una propuesta pedagógica para la construcción de paz. Cuadernos de Administración, 28(51), 159-187.

Habermas, J. (1989). Teoría de la Acción-Comunicativa, vol. 2, Buenos Aires-Argentina, Taurus.
Hoyos, G. \& Vargas, G. (1997). La teoría de la acción comunicativa como nuevo paradigma de investigación en las ciencias sociales: Las ciencias de la discusión, Bogotá-Colombia.

Latorre, M. (2016). Aprendizaje Significativo y Funcional Aplicación en el aula [Mensaje en un blog]. Recuperado de http://marinolatorre.umch.edu.pe/aprendizaje-significativo-y-funcional/

Ministerio de Educación Nacional, República de Colombia. (2019). Formar para la ciudadanía, ¡Si es posible! Bogotá-Colombia: Espantapájaros Taller. Obtenido de https://www.mineducacion.gov.co/1621/articles-75768_ archivo_pdf.pdf

Miranda-Medina, C. \& Gorjón, F. (2018). Mediación, su valor intangible y efectos operativos. Una visión integradora de los métodos alternos de solución de conflictos. Comunitania, 15, 276 - 280.

Miranda-Medina, C. \& Rodríguez-Burgos, K. (2017). Las publicaciones científicas en investigación y educación latinoamericana. Educación y Humanismo, 19 (32), 10-17. doi:https://doi.org/. https://doi. org/10.17081/eduhum.19.32.2638.

Miranda-Medina, C., Ruiz-Gómez, G., Rodríguez-Burgos, K. \& Gorjón, F. (2020). School mediation a tool against school violence. En K. Rodríguez-Burgos (coord.), School violence radiography (pág. 119-140). Barranquilla: Ediciones Universidad Simón Bolívar.

Muñoz, F., \& López Martínez, M. (2004). Historia de la Paz. En B. Molina Rueda, \& F. Muñoz, Manual de Paz y Conflictos. Granada: Eirene.

Oliveros Zambrano, A., Pulido González, E. F., \& Rincón Pinilla, Y. P. (2017). El cómic como mediación pedagógica para la construcción de una cultura de paz y reconciliación en el grado sexto del Instituto San Bernardo de La Salle.

Palos Rodríguez, J. (2010). Programa de Educación en valores. Obtenido de Organización de los Estados Iberoamericanos para la Educación, la Ciencia y la Tecnología: http://www.oei.es/valores2/palos1.htm

Perez de Guzman Puya, M.V. (2009). La mediación en los 
centros educativos. El educador social como mediador. Bordon. Revista de Pedagogía.

Pérez Gómez, A. (2008). ¿Competencias o pensamiento práctico? La construcción de los significados de representación y de acción. Madrid: Morata.

Pérez Sánchez, L. y Beltrán Llera, J. (2014). Estrategias de aprendizaje: Función y diagnóstico en el aprendizaje del adolescente. Padres y Maestros, 358, 34-38. https:// doi.org/10.14422/pym.v0i358.4086.

Riveros, X. (2009). Constructivismo: elementos conceptuales. Santiago de Chile: Universidad de Chile.

Rodríguez-Burgos, K. \& Gorjón, E. (2014). Grupos de enfoque. En K. Sáenz \& Tamez, Métodos y técnicas cualitativas y cuantitativas aplicables a la investigación en ciencias sociales (págs. 32-44). Mexico: Tirant Humanidades.

Rodríguez-Burgos, K. \& Miranda-Medina, C. (2020). School violence radiography conclusions. En K. Rodríguez-Burgos, School violence radiography (pág. 141-153). Barranquilla: Ediciones Universidad Simón Bolívar.

Rodríguez-Burgos, K. (2012). Investigación cuantitativa: Diseño, técnicas, muestreo y análisis cuantitativo. En K. Sáenz, F. Gorjón, M. Gonzalo \& Díaz, Metodología para investigaciones de alto impacto en las Ciencias Sociales y Jurídicas. México: Dykinson.

Rodríguez-Burgos, K., Morcote, O., Miranda-Medina, C. \& Gallegos, M. (2020). Studies, causes, perception, measurement and results of school violence. En K. Rodríguez-Burgos (coord.), School violence radiography (pág. 83-117). Barranquilla: Ediciones Universidad Simón Bolívar.

Sáenz, K. \& Rodríguez-Burgos, K. (2010). La promoción de la participación ciudadana. En K. Saenz López, \& M. Estrada Camargo, Elecciones, gobierno y gobernabilidad (págs. 149-176). México: Instituto Federal Electoral.

Suárez, A. A., \& Pimiento, A. B. (2018). La educación en cultura de paz. Herramienta de construcción de paz en el postconflicto. Reflexión política, 20(40), 5.

Vargas Gómez, D. F. (2019). Aprendizaje Significativo des- de el Cómic: Una Propuesta de Innovación para la Enseñanza de la Historia Bipartidista.

Vázquez Gutiérrez, R. L., (2015). Mediación educativa como instrumento de pacificación social. La multidisciplinariedad de la mediación y sus ámbitos de aplicación. Edita: Tirant Lo Blanch, México.

Velasco Suárez, M., Ordoñez Acuña, B., Dávila, M. y Rotter Hernández; L. (2012). Una educación para la paz. Programa de trabajo para niños preescolares. México: Trillas.

Weber, M. (1977). Economía y sociedad. México: Fondo de cultura económica.

\section{Lourdes Albor-Chadid}

Doctora en Métodos Alternos de Solución de Conflictos; Psicóloga. Especialista en Psicología Clínica, Magister en Desarrollo Educativo y Social. Profesora-Investigadora Grupo Estudios de Género, Familia y Sociedad, Categoría A1, Universidad Simón Bolívar, Barranquilla, Colombia.lalbor1@unisimonbolivar.edu. CO 